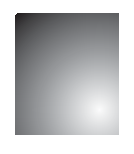

\title{
Análise Bibliométrica da Contribuição de Marketing PARA OUTRAS CIÊNCIAS
}

\author{
Bibliometric Analysis of the Contribution of Marketing to other \\ Sciences
}

\section{Carlos Alberto Vargas Rossi}

Doutor em Administração pela Universidade de São Paulo - USP. Professor Titular da Universidade Federal do Rio Grande do Sul. Porto Alegre, RS. Brasil. E-mail: cavrossi@ea.ufrgs.br

\section{Luiza Venzke Bortoli}

Doutoranda em Administração pela Universidade Federal do Rio Grande do Sul - UFRGS. Professora do Instituto Federal de Educação, Ciência e Tecnologia do Rio Grande do Sul - IFRS. Feliz, RS. Brasil. E-mail: luizabortoli@gmail.com

\section{Rodrigo Bisognin Castilhos}

Doutorando em Administração pela Universidade Federal do Rio Grande do Sul - UFRGS. Professor Assistente da Universidade do Vale do Rio dos Sinos. São Leopoldo, RS. Brasil.E-mail: rbcastilhos@gmail.com

\section{Resumo}

O presente estudo busca analisar a contribuição da disciplina de marketing para outras áreas de conhecimento. Para tanto, foi realizado um estudo bibliométrico, verificando a presença, por meio de citações, das revistas de maior relevância da área de marketing nas principais publicações de outras áreas correlatas. Os resultados evidenciam um número apenas limitado de citações aos periódicos de marketing selecionados. Dentre as áreas analisadas, apenas gestão de negócios recebe significativa influência dos estudos de marketing. Entre os temas mais influentes, verificou-se a presença constante daqueles ligados a métodos de pesquisa e à subárea do comportamento do consumidor. Diante desses resultados, é possível refletir sobre a necessidade de a área buscar maior relevância junto às demais ciências sociais e humanas, uma vez que se ocupa de domínios relevantes das relações sociais e de mercado na sociedade contemporânea.

Palavras-chaves: Marketing. Escopo de Marketing. Análise Bibliométrica.

\section{Abstract}

This study aims to analyze the contribution of the Marketing discipline to other areas of knowledge. For this purpose, a bibliometric study was conducted, which, through citations, verified the presence of the most relevant marketing journals in major publications from other areas. Results indicate that only the area of Management receives significant influence of marketing studies. The most influential articles are within the scope of research methods and consumer behavior, suggesting a limited influence of Marketing in other areas. After analyzing results, we try to reflect on the need to make Marketing a more relevant area to other social and human sciences, since it deals with important domains of social and market relations in contemporary society.

Keywords: Marketing. Marketing Scope. Bibliometric Analysis. 


\section{INTRODUÇÃo}

A disciplina do marketing jamais caminhou solitariamente. Tendo se apropriado, inicialmente, dos conhecimentos oriundos da economia e da própria administração, o seu desenvolvimento passou pelo aporte teórico de disciplinas como a estatística, a psicologia, a sociologia e a antropologia (BARTELS, 1988; WILKIE; MOORE, 2003). Contudo, desde seus primórdios, a disciplina vem ampliando a fronteira do conhecimento nos assuntos dos quais se ocupa. Levando-se em conta a quantidade de programas de pós-graduação stricto sensu, de encontros e periódicos científicos, a produção acadêmica de marketing pode ser considerada vultosa e variada, mesmo que baseada significativamente no conhecimento de outras ciências sociais (PIETERS; BAUMGARTNER, 2002). Então, qual é o tamanho da contribuição do marketing para as disciplinas que influenciaram e seguem influenciando a área? Quais são os temas de marketing que estão presentes nas diferentes áreas? Com que intensidade isso ocorre?

Para encaminhar uma discussão acerca desses questionamentos, a presente pesquisa visa analisar a contribuição da disciplina de marketing para outras áreas de conhecimento. Para tanto, é realizado um estudo bibliométrico (MORAN et al., 2010; WALTER; ROCHA, 2011; FERREIRA et al., 2012; RIBEIRO et al., 2012), verificando a presença, por meio de citações, das revistas de maior relevância da área de marketing nas principais publicações de outras áreas de conhecimento, tais como: negócios, comunicação, psicologia, economia e finanças, sociologia e antropologia. A referência a um artigo de um periódico indica que o artigo citado é uma parte de conhecimento relevante e importante para outros acadêmicos (PIETERS; BAUMGARTNER, 2002; FERREIRA et al., 2012). Por meio das citações, há legitimação tanto do artigo escrito como do citado (SMALL, 1978). Busca-se, com isso, verificar se o conhecimento científico de marketing é utilizado além de suas fronteiras, oferecendo uma medida da importância da área para o avanço do conhecimento científico nas demais ciências sociais.

Para a seleção dos periódicos a serem consultados, utilizou-se o fator de impacto de cinco anos do Journal of Citation Reports, por meio do qual se certifica a qualidade deles e a relevância que eles têm para suas áreas. Após a seleção dos periódicos, foram analisados cinco anos e meio de publicações em cada periódico, verificando, artigo por artigo, a quantidade de trabalhos de marketing citados, o números de vezes que os artigos foram citados dentro dos textos, bem como informações sobre os autores, ano e periódico de origem da citação. Dessa forma, entende-se que seja possível mapear o alcance da influência da área de marketing em outras áreas do conhecimento.

O restante do artigo está construído de forma a colocar em perspectiva a relação da área de marketing com outras áreas de conhecimento, para então analisar a possível contribuição da primeira para as últimas. Primeiramente, é feita uma breve reconstituição acerca da natureza interdisciplinar e do escopo da disciplina; após, são analisados aspectos atinentes à relevância da área de marketing. Em terceiro lugar, é apresentada a proposta metodológica, com uma breve discussão acerca dos estudos bibliométricos e o detalhamento dos procedimentos adotados. Em quarto, são apresentados e discutidos os resultados para, finalmente, serem tecidas as considerações finais.

Entende-se que a disciplina do marketing é produtora de um conhecimento científico com um ponto de vista particular sobre uma ampla gama de fenômenos, tais como estratégias empresariais, dinâmicas de mercado e sociedade de consumo (HUNT, 1976; JONES; SHAW; McLEAN, 2010), com potencial para contribuir de maneira consistente com as demais ciências sociais correlatas, puras e aplicadas. Com a apresentação de um instantâneo sobre a relevância da área, o presente artigo pretende oferecer um ponto de partida para a sua problematização e para a identificação de pontos de intersecção com outras disciplinas, na busca pelo incremento da influência do conhecimento gerado em marketing para além de suas fronteiras.

\section{Sobre a Natureza Interdisciplinar e o Escopo de Marketing}

A disciplina do marketing surgiu no final século XIX nos Estados Unidos, voltada para o estudo dos processos de distribuição dos bens (BARTELS, 1988). Foi, porém, com o desenvolvimento da produção em massa e o estabelecimento do capitalismo monopolístico, no início do século $\mathrm{XX}$, que o marketing consolidou-se como função gerencial e como disciplina no contexto 
americano, lidando principalmente com os sistemas distributivos (DHOLAKIA; FIRAT; BAGOZZI, 1980). Com o decorrer do tempo e as transformações sociais do século XX, a disciplina passa de uma visão centrada na empresa e em seus produtos - visão meramente gerencial - para uma ótica voltada para os clientes e para o mercado, entendendo o marketing como um processo social e econômico (FULLERTON, 1988; KEITH, 1960; VARGO; LUSCH, 2004). Nos primeiros anos de seu desenvolvimento foram utilizados, principalmente, conceitos da economia, para lidar com a demanda e com as questões de distribuição (BARTELS, 1988). A partir da década de 1950 , o marketing passa a incorporar o conhecimento das ciências quantitativas e comportamentais, notadamente a estatística e a psicologia (WILKIE; MOORE, 2003), no sentido de entender conjuntos de consumidores, de modo a exercer influência sobre eles. A partir de então, a disciplina se desenvolveu com base na apropriação de conhecimentos de diversas ciências humanas e sociais, para compreender os fenômenos dos quais se ocupa. Mas, que fenômenos são esses? Qual é o escopo da disciplina? De onde vêm as principais contribuições para seu progresso?

Analisando as escolas do pensamento de marketing, é possível verificar quais são esses fenômenos e compreender as diferentes fontes de influência que pairam sobre a disciplina. A tipologia desenvolvida por Shaw e Jones (2005) e ampliada por Jones, Shaw e McLean (2010) explicita as principais questões abordadas, o foco de análise, os conceitos-chave e as teorias de cada escola: a escola de "funções de marketing" aborda as principais atividades que compõem o processo de marketing, procurando explicar os papéis dos diferentes agentes envolvidos ao longo desse processo. A escola de "produtos" compreende a classificação dos diferentes tipos de bens, de acordo com suas características e com o tipo de destinação que eles possuem no mercado, também com influência da economia. A escola de "instituições de marketing" trata de canais de distribuição, incluindo definições sobre os intermediários de marketing e suas funções no processo. A "escola de administração de marketing" trabalha com questões de nível micro, incluindo composto de marketing, orientação para o consumidor e decisões estratégicas de marketing, sob a perspectiva dos produtores. A construção do conhecimento nessas quatro primeiras escolas se dá sob uma clara influência das teorias econômicas e da área de negócios. A escola de "sistemas de marketing" procura entender a área e os mercados sob a perspectiva sistêmico-funcionalista, buscando compreender os diferentes atores que desempenham papéis em um mercado, tanto em nível macro (sociedade) como em nível micro (lares). A "escola de macromarketing" envolve as questões de troca em um nível macro, abordando o impacto das ações de marketing na sociedade e vice-versa. A "escola de troca" inclui questões sobre a negociação que ocorre entre as partes envolvidas. Essas escolas se valem do aporte teórico das áreas correlatas às organizações, mas também da sociologia, ética e ciência política, além da economia, no caso específico da "escola de troca". Já a escola de "história do marketing" é menos uma escola e mais um conjunto de esforços para mapear o desenvolvimento histórico da área de marketing do ponto de vista gerencial e acadêmico. Por fim, a escola do "comportamento do consumidor", que se caracteriza por ser a mais autônoma e eclética das correntes, focando nos aspectos que envolvem o processo de compra e consumo dos indivíduos. Nesta escola, verifica-se a maior amplitude de influências, desde a economia até a sociologia e antropologia, passando pela psicologia, possivelmente a principal influência desta escola.

A análise conjunta das escolas dá conta da amplitude de temas e influências abarcados pelo campo do marketing em sua produção de conhecimento. Desde a contenda entre Kotler e Levy (1969a, 1969b) e Luck (1969), percebe-se que a ampliação do escopo da disciplina foi muito mais longe do que esses autores originalmente imaginavam. Em outro esforço de classificação, Hunt (1976) desenvolveu um modelo conceitual que circunscreve o escopo da disciplina a três categorias dicotômicas: setor lucrativo versus não lucrativo; micro versus macro; e, positivo versus normativo, que formam oito domínios possíveis cobertos pela produção de conhecimento em marketing: (1) lucrativo-micropositivo, envolvendo pesquisas e formulação de teorias relacionadas ao comportamento do consumidor individual e ao comportamento gerencial das firmas; (2) lucrativo-micronormativo, envolvendo pesquisas e modelos que procuram prescrever como as firmas devem gerenciar seu mix de marketing, elaborar estratégias, desenhar seus departamentos, entre outros; 
(3) lucrativo-macropositivo, que compreende pesquisas sobre o comportamento agregado dos consumidores, sobre aspectos legais do mercado, sobre os sistemas de marketing e sua eficiência, justiça e conceito, entre outros; (4) lucrativo-macronormativo, que endereça questões e modelos normativos relativos ao modo como o mercado pode ser mais eficiente, sobre os limites da propaganda, sobre o quanto é desejável estimular a demanda ou regular o mercado, entre outros; (5) não lucrativo-micropositivo, envolvendo pesquisas referentes ao comportamento do consumidor de bens públicos e às práticas de marketing de organizações públicas; (6) não lucrativo-micronormativo, envolvendo pesquisas e modelos sobre como as organizações públicas devem planejar e gerenciar a sua oferta; (7) não lucrativo-macropositivo, que compreende pesquisas relacionadas à estrutura institucional dos bens públicos, à influência da propaganda em eleições, à eficiência dos bens públicos, entre outros; e, (8) não lucrativo-macronormativo, que se refere a modelos sobre se a demanda por bens públicos deve ser estimulada, sobre a sociedade permitir que políticos sejam "vendidos", sobre os modelos de propaganda política socialmente desejáveis, entre outros.

O que se evidencia tanto na análise das escolas quanto na dos oito domínios é um esforço deliberado dos acadêmicos em abranger aspectos que vão além das trocas economicamente constituídas, ocupando-se de questões que, em um extremo encontram-se nas transações ordinárias entre as empresas e seus clientes e, em outro extremo, nas relações entre empresas, governos, cidadãos e políticas públicas, dentro do que se rotulou de marketing social (ANDREASEN, 2003; HASTING; SAREN, 2003). Essa diversidade de temas, interesses e influências acabou contribuindo para o amadurecimento de um campo acadêmico independente, que rapidamente fragmentou-se e especializou-se em subáreas, perdendo, aos poucos, sua conexão com a disciplina mais ampla de administração e negócios (BAUMGARTNER; PIETERS, 2003). Tal ampliação trouxe benefícios inegáveis para a área no que diz respeito à difusão das ferramentas e tecnologias de marketing em nível gerencial e à própria ampliação do campo de atuação dos profissionais, porém até que ponto essa ampliação contribui para a relevância acadêmica da área? Em que medida as ciências que influenciam a construção de conhecimento em marke- ting são, também, influenciadas pelo conhecimento por ela produzido? Existem áreas com as quais o diálogo é mais profícuo? Nas próximas seções procurou-se abordar esses e outros questionamentos.

\section{Sobre a Relevância Acadêmica da Área de Marketing}

A reflexão sobre a relevância da área de marketing envolve discussões sobre a lacuna entre teoria e prática, a ênfase em metodologia e a forma institucionalizada de divulgação do conhecimento produzido no campo, entre outras. Já do ponto de vista das contribuições para o conhecimento fora das fronteiras da área específica, as discussões envolvem o entendimento da natureza e da intensidade da contribuição de marketing para outras áreas de conhecimento.

Se a importância das tecnologias de marketing passa a ser crucial para as organizações no atual contexto competitivo, o mesmo não se pode dizer da relevância dos conhecimentos acadêmicos de marketing para realização dessas tecnologias no dia a dia dessas empresas. Com efeito, a ainda existente - e talvez crescente - lacuna entre teoria e prática (MCALISTER, 2005) é tomada como uma ameaça à relevância aplicada da disciplina. Com isso, diversos aspectos importantes de marketing seriam apropriados por outras áreas nas organizações e seu papel se limitaria, em muitos casos, a um mero suporte à área de vendas (SHETH; SISODIA, 2005), paradoxalmente reduzindo o escopo de atuação dos praticantes. Porém, a utilidade prática passa pela produção de conhecimento. Nesse sentido, Hunt (2002) considera que, sendo marketing uma disciplina universitária e quase profissional, as pesquisas acadêmicas da área deveriam ter em conta as necessidades de seus clientes, que, em última instância, é a "[...] necessidade da sociedade por produtose serviços de alta qualidade que são precificados razoavelmente, promovidos responsavelmente e disponíveis convenientemente". (HUNT, 2002, p. 57)

Para MacInnis (2005), um dos aspectos capazes de minar a relevância da área é o foco em metodologia, responsável por uma confusão nos critérios de relevância e qualidade das pesquisas, bem como por uma limitação na própria agenda de pesquisas de acordo com seu enquadramento nas predileções metodológi- 
cas do campo. No limite, a categorização pelo método bifurca a área em "campos", que acabam se vendo mutuamente como irrelevantes ou como adversários.

Relacionado aos dois tópicos já discutidos, o sistema de publicações científicas é alvo de críticas de alguns acadêmicos. Para McAlister (2005), a já citada divisão da área em campos (MacInnis, 2005), cada qual com sua definição do que seria uma pesquisa adequada, acaba por contaminar as principais publicações científicas, "controladas" por grupos pertencentes a diferentes campos. Isso levaria a uma perda de foco $e$ de critérios para publicação, que valoriza mais as regras rígidas sobre o que viria a ser uma "pesquisa válida", deixando de lado perguntas primordiais, como: "Este artigo fornece uma nova visão? Este artigo 'não está errado'?" (MCALISTER, 2005, p. 16). No limite, essa perda de foco leva a não publicação de contribuições originais para o conhecimento em marketing (HUNT, 2002).

Se há restrições de parte de alguns acadêmicos em relação à relevância da disciplina para a própria disciplina, quando se olha para a possibilidade de contribuição para outras áreas, essas dúvidas são intensificadas. Dentre os primeiros autores a chamar a atenção para a falta de interesse das outras disciplinas estão Day e Montgomery (1999), que afirmam de forma contundente que os acadêmicos de fora da área de marketing prestam pouca atenção ao conhecimento produzido por ela.

Essa falta de interesse fica mais evidente na análise de citações conduzida por Pieters e Baumgartner (2002). Os autores analisaram a comunicação interdisciplinar entre economia e outras áreas, como antropologia, ciência política, psicologia, sociologia e cinco áreas de negócios, incluindo contabilidade, finanças, administração, marketing e sistemas de informação/ operações. Foram analisadas as citações cruzadas dos principais periódicos desses campos durante 1995 e 1997. Foi encontrado que, para as disciplinas pesquisadas, a economia surge como uma fonte principal de conhecimento, embora ela própria faça pouco uso do conhecimento de suas "disciplinas irmãs". Apenas $10 \%$ das citações dos cinco principais periódicos de economia no período analisado são interdisciplinares, estando a maioria vinculada a finanças, sendo essa a única disciplina com a qual economia possui relações de citações mútuas substanciais. Os cinco periódicos de economia selecionados não fizeram nenhuma citação às áreas de administração, marketing, antropologia e psicologia durante os anos considerados. Comparada a finanças, contabilidade tem suas raízes menos influenciadas pela economia. Já marketing, sistemas de informação/operações e administração absorvem ainda menos conhecimento da economia e estão baseados, significativamente, no conhecimento de outras ciências sociais e demais áreas de administração para formar seus próprios campos de estudo, o que não se reflete na existência significativa de citações de marketing nas outras áreas.

\section{Análise de Citações}

No meio acadêmico, a produtividade de um autor é medida por suas publicações. Tais publicações são a forma consagrada pela academia para relatar a produção de conhecimento em grande parte dos campos científicos. Para serem legitimadas, essas obras devem ser publicadas em um conjunto de periódicos, que adotam pressupostos peculiares de avaliação de qualidade do trabalho, conferindo legitimidade a eles e formando a reputação desse conjunto de periódicos. Dentre os pressupostos acima está o de que um trabalho de qualidade ergue-se a partir de um arcabouço teórico que dá sustentação para a condução da obra. É na construção desse arcabouço que os acadêmicos fazem uso de pesquisas anteriormente realizadas, preferencialmente publicadas nesses mesmos periódicos. A utilização de diferentes trabalhos em um artigo materializa-se na forma de citações, que, ao mesmo tempo, legitimam o artigo que está sendo escrito $e$ os artigos nele citados, fechando um ciclo de criação de significado desse artigo (SMALL, 1987). Assim, o ato de citar um artigo denota: (1) valorização de seu conteúdo pelo pesquisador; (2) influência desse conteúdo na pessoa do pesquisador; (3) significância desse conteúdo para a construção do conhecimento em questão. (SMALL, 1987; FERREIRA et al., 2012)

É a partir dessa desnaturalização da citação que se pode afirmar que a relevância científica de uma obra, autor ou área para determinado campo do conhecimento somente pode ser julgada retrospectivamente, a partir da análise da intensidade e da forma com que tal obra, autor ou área foram utilizados (ANDERSON, 
2006). Dentre as formas para acessar essa relevância estão os estudos bibliométricos, por meio dos quais é possível mensurar a utilização de determinada referência em diferentes níveis, incluindo (ANDERSON, 2006): (1) exame da produtividade de um autor ou de determinado departamento; (2) fornecimento de uma medida de qualidade para fazer um ranking de periódicos; (3) identificação dos artigos mais frequentemente citados em uma área; (4) identificação de cocitações, de modo a traçar a estrutura de um dado campo.

A utilização de estudos bibliométricos como forma de criação de sentido acerca do corpo de conhecimento gerado em diferentes áreas vem crescendo no contexto brasileiro (MORAN et al., 2010; WALTER; ROCHA, 2011; FERREIRA et al., 2012; RIBEIRO et al., 2012). No marketing, diversas pesquisas têm utilizado a análise de citações. Tais pesquisas incluem: análise da produtividade dos pesquisadores no contexto brasileiro (VIEIRA, 2010); análise dos temas mais pesquisados no principal periódico de comportamento do consumidor (ROSSI, 2008); análise dos modos de apropriação do pensamento do sociólogo Pierre Bourdieu no campo, no contexto brasileiro (CASTILHOS, 2007); análise da influência interna dos principais periódicos de marketing e correlatos em um período de 30 anos (BAUMGARTNER; PIETERS, 2003). Juntos, esses trabalhos constituem uma espécie de inventário da produção acadêmica, dos principais autores e das influências que o campo recebe e emite no caminho da produção de conhecimento científico relevante. É com a preocupação de contribuir para esse inventário que a presente análise de citações foi levada a cabo.

\section{Procedimentos}

Para acessar a influência do marketing sobre outras áreas do conhecimento, optou-se por realizar uma análise das citações dos principais periódicos de marketing nos principais periódicos das demais áreas. A seleção das áreas ocorreu a partir da proximidade que cada uma possui com o marketing. Nota-se que o diálogo com outros campos ocorre em diferentes níveis de influências mútuas, conforme verificado na breve análise do escopo da disciplina: ( $1^{\circ}$ nível) áreas de gestão de negócios e de comunicação como as mais próximas, sendo a primeira, a área de conhecimento na qual está vinculada a maior parte dos cursos de marketing em todo o mundo, e a segunda, uma área em que cada vez mais disciplinas de marketing são incluídas nos currículos; ( $2^{\circ}$ nível) área de economia e de finanças, sendo a primeira a ciência-mãe do marketing, e a segunda, uma área fronteiriça entre a gestão de negócios e a economia; e ( $3^{\circ}$ nível) áreas de psicologia, antropologia e sociologia, consideradas ciências mais puras, cuja influência concentra-se especialmente na escola de comportamento do consumidor.

Para a seleção dos principais periódicos, foi utilizado o fator de impacto de cinco anos, do Journal of Citation Reports ${ }^{1}$. Ao passo que o fator de impacto simples dá conta da relevância de um determinado periódico durante dois anos, o fator de impacto de cinco anos mostra-se mais adequado para a análise da influência de longo prazo de determinado periódico, pois é menos influenciado pelas edições especiais sobre um tema, por estratégias de autorreferência, pela existência de um artigo cuja influência seja acima da média, entre outros aspectos que podem inflacionar o fator de impacto simples.

No primeiro nível de influência das áreas estão negócios e comunicação. Os periódicos de negócios e seus fatores de impacto são: Academy of Management Review (9,531), Academy of Management Journal $(9,263)$, MIS Quarterly $(9,208)$, Strategic Management Journal $(6,931)$ e Administrative Science Quarterly $(6,216)$. Os periódicos de comunicação e seus fatores de impacto são os seguintes: Public Opinion Quarterly (3,301), Journal of Communication (2,765), Human Communication Research $(2,482)$, Cyberpsychology Behavior $(2,472)$ e Journal of Health Communication $(2,434)$.

No segundo nível de influência das áreas estão economia e finanças, cujos periódicos são: Journal of Economic Literature (8,922), Quarterly Journal of Economics (8,171), Journal of Political Economy $(6,924)$, Journal of Finance $(6,536)$ e Journal of Financial Economics $(5,675)$.

No terceiro nível de análise das áreas de maior impacto no campo do marketing há psicologia, antropologia e sociologia. Para a psicologia, o Journal of Citation Reports apresenta listagens de psicologia aplicada, psicologia experimental, psicologia multi- 
disciplinar e psicologia social, os quais foram considerados em conjunto, resultando na seguinte listagem: Annual Review of Psychology $(21,025)$, Psychological Bulletin (19,160), Trends in Cognitive Sciences (15,591), Psychological Review (11,582) e Advances in Experimental Social Psychology (10,733). Para a antropologia, os principais periódicos são: Journal of Human Evolution (4,077), Evolutionary Anthropology (3,864), Annual Review of Anthropology $(3,559)$, Social Networks $(3,328)$ e Current Anthropology $(3,113)$. Verifica-se que entre os principais periódicos de sociologia, eliminou-se Social Networks, por já constar na lista de antropologia, e Cornell Hotel and Restaurant Administration Quarterly, que não faz parte do escopo da área. Este último é a principal publicação das indústrias de hospitalidade e serviços de alimentação, sendo destinado a avanços nos princípios de gestão relevantes para o campo, tendo aparecido na listagem, possivelmente, por erros nos parâmetros do próprio Journal of Citation Reports no momento da consulta. Foram inclusos, portanto, os dois próximos periódicos da área, de acordo com o fator de impacto utilizado. Os periódicos de sociologia analisados são: Annual Review of Sociology (5,953), American Sociological Review (5,578), American Journal of Sociology $(5,411)$, Sociological Methods and Research $(3,596)$ e Journal of Marriage and Family $(2,957)$.

Para determinar os principais periódicos de marketing, foram adotados os mesmos critérios utilizados para as demais áreas. Tendo em vista que o Journal of Citations Reports não apresenta uma classificação única de marketing, foram selecionadas as dez principais publicações da área presentes na classificação de negócios, as quais são: Journal of Marketing $(8,520)$, Journal of Retailing (5,181), Journal of Consumer Research (5,030), Journal of Marketing Research $(4,768)$, Journal of Consumer Psychology (4,559), Marketing Science (4,251), Journal of Interactive Marketing $(4,021)$, Journal of Service Research $(3,973)$, Journal of Consumer Affairs $(2,168)$ e Journal of Public Policy \& Marketing $(2,157)$.

Após a seleção dos periódicos, procedeu-se a busca pelas referências dos principais periódicos de marketing em cada um dos periódicos das outras áreas em todos os números e volumes, durante um período cinco anos e meio, de 2006 a 2011². Para cada número de revista foi realizada uma busca que obedeceu aos seguintes passos: (1) busca pela presença de citação dos periódicos de marketing selecionados dentro do número da revista em questão; (2) download de todos os artigos citando os periódicos de marketing, e; (3) análise de cada artigo e alimentação da base de dados em formato Excel com as seguintes informações: área, título, ano, volume e número do periódico; título e autores do artigo que faz a citação de marketing; autores, ano, título do artigo e do periódico citado, além do número de vezes que o artigo foi citado ao longo do texto, o que permite ter a noção da intensidade individual e agregada da influência dos artigos de marketing.

Após a coleta dos dados, foram conduzidas análises de frequência e cruzamentos por ano, área do conhecimento, quantidade de citações e autores mais citados. Os resultados permitem encaminhar os seguintes pontos: (1) áreas que mais citam os principais periódicos de marketing; (2) periódicos mais citados no geral e por área; e (3) média de vezes, no geral e por área, que os artigos de marketing são citados. As seguintes considerações são destinadas à apresentação e à discussão dos resultados dessa pesquisa.

\section{Resultados e Discussão}

A Tabela 1 apresenta a quantidade e o percentual relativo de artigos que fazem referência a textos dos dez principais periódicos de marketing. Doravante o termo "referência" será utilizado para designar os artigos de marketing que aparecem na seção references, ao final de cada artigo, ao passo que o termo "citação" será utilizado para designar a situação em que um artigo é efetivamente citado ao longo de um texto. Além disso, tendo em vista que cada artigo de uma área analisada pode fazer diversas citações de um único artigo de marketing, foi feita uma contagem do total de citações a artigos de marketing. Acrescenta-se, também, a média de citações de marketing por artigo, a qual é obtida pela divisão do total de citações pelo número de artigos encontrados que citam marketing. 
Carlos Alberto Vargas Rossi • Luiza Venzke Bortoli • Rodrigo Bisognin Castilhos

Tabela 1: Artigos que referenciam marketing e citações aos artigos de marketing

\begin{tabular}{|c|c|c|c|c|c|}
\hline \multirow[t]{2}{*}{ ÁREAS } & \multicolumn{2}{|c|}{$\begin{array}{c}\text { ARTIGOS QUE REFERENCIAM } \\
\text { MARKETING }\end{array}$} & \multicolumn{2}{|c|}{$\begin{array}{c}\text { TOTAL DE CITAÇõES A ARTIGOS DE } \\
\text { MARKETING }\end{array}$} & \multirow{2}{*}{$\begin{array}{l}\text { MÉdIA DE CITAÇÕES DE MARKETING } \\
\text { POR ARTIGO ONDE MARKETING É } \\
\text { REFERIDO }\end{array}$} \\
\hline & $\mathbf{N}$ & $\%$ & $\mathbf{N}$ & $\%$ & \\
\hline Negócios & 369 & 59 & 2.585 & 69 & 7,01 \\
\hline Comunicação & 148 & 24 & 815 & 22 & 5,51 \\
\hline Psicologia & 48 & 8 & 212 & 6 & 4,42 \\
\hline Economia e Finanças & 28 & 4 & 70 & 2 & 2,50 \\
\hline Sociologia & 26 & 4 & 68 & 2 & 2,62 \\
\hline Antropologia & 10 & 2 & 19 & 1 & 1,90 \\
\hline Total & 629 & 100 & 3.769 & 100 & 3,99 \\
\hline
\end{tabular}

Fonte: Elaborada pelos autores deste artigo

Os dados obtidos confirmam que as áreas de negócios e comunicação são as mais próximas ao marketing dentro do período analisado. No entanto, nota-se que a área de psicologia possui maior número de referências ao marketing do que economia e finanças. Isso pode ser um indício do destaque dos estudos de comportamento do consumidor no marketing, o qual cria uma proximidade com a psicologia. Verifica-se, assim, a existência de níveis de influência relacionados a intensidade de citações de artigos de marketing diferentes daqueles definidos a priori, onde no primeiro nível encontra-se a área de negócios (59\% das referências e $69 \%$ das citações encontradas), no segundo nível a área de comunicação ( $24 \%$ das referências e $22 \%$ das citações) e no terceiro nível as demais áreas ( $18 \%$ das referências e $11 \%$ das citações). Por fim, os resultados dessa análise geral vão de encontro aos argumentos de Baumgartner e Pieters (2003) sobre o distanciamento crescente de marketing das áreas de negócios, evidenciando, ao contrário, a existência de temas da primeira que alimentam discussões da segunda.

Para averiguar a contribuição do marketing ao longo do tempo, foi analisada a variação da quantidade de artigos por área que referenciaram as publicações de marketing entre 2006 e 2010. Negócios e comunicação possuem as maiores variações, com médias anuais de 66 e 29 artigos que referenciaram marketing e desvios -padrão de 4,24 e 4,77, respectivamente. Em negócios houve aumento de referências a marketing entre 2006 e 2009, com redução em 2010. Em comunicação houve decréscimo entre 2006 e 2009 e aumento em 2010. Economia e finanças mantiveram-se estáveis com 6 artigos referenciando publicações de marketing a cada ano, exceto em 2007, quando houve apenas um. Psicologia, sociologia e antropologia apresentaram médias de 8, 4,6 e 1,6, respectivamente, e pequena variação na quantidade de referências a marketing ao longo do tempo, com desvios-padrão próximo a 1 (1,10, 1,02 e 1,36, respectivamente).

A fim de verificar a atualidade das referências à área de marketing, analisou-se a relação entre a data de publicação dos artigos de cada área em relação à data de publicação dos artigos de marketing referenciados. Verificou-se que a idade média dos artigos referenciados varia de 14 a 16 anos para todas as áreas, exceto para psicologia, que apresentou uma média de 8,4 anos. Os achados podem indicar duas tendências a ser confirmadas na análise individual das áreas: (1) ênfase em artigos tidos como clássicos da área de marketing, especialmente os artigos de metodologia, largamente citados; e (2) ênfase em assuntos que tiveram o auge de sua produção acadêmica na primeira metade dos anos de 1990.

Outro dado importante refere-se à quantidade de diferentes artigos referenciados (67\%), bem como o número total de referências sem considerar a repetição de um mesmo artigo. Em todas as áreas há maior frequência de artigos diferentes do que repetidos, indicando que pesquisadores das áreas analisadas fazem uso de artigos variados de marketing para suas pesquisas.

Com uma visão geral do impacto do marketing em outras áreas, parte-se para um estudo de cada área. Para isso, foram analisados os seguintes pontos: 
número de diferentes artigos de marketing referenciados em cada área de acordo com o periódico, número total de artigos de marketing referenciados por área de acordo com o periódico, artigos de marketing mais referenciados por área e autores de marketing com maior número de artigos diferentes referenciados por outras áreas.

\subsection{A Influência de Marketing na Área de Negócios}

Entre as áreas analisadas, a de negócios foi a que apresentou maior proximidade com o marketing. É importante verificar em quais periódicos essa relação é mais frequente. Para isso, observa-se a Tabela 2 , a qual apresenta a quantidade $e$ a frequência de diferentes artigos de marketing referenciados, bem como o número total de referências de acordo com os periódicos de marketing.

Tabela 2: Quantidade de artigos de marketing referenciados em negócios

\begin{tabular}{|c|c|c|c|c|}
\hline \multirow{2}{*}{$\begin{array}{c}\text { PeRiódicos de MARKETING } \\
\text { (RANKING FI } 5 \text { ANOs) }\end{array}$} & \multicolumn{2}{|c|}{$\begin{array}{c}\text { Artigos } \\
\text { DifERENTES }\end{array}$} & \multicolumn{2}{|c|}{ TOTAL } \\
\hline & $\mathbf{N}$ & $\%$ & $\mathbf{N}$ & $\%$ \\
\hline Journal of Marketing (1) & 255 & 30 & 474 & 33 \\
\hline $\begin{array}{l}\text { Journal of Marketing } \\
\text { Research (4) }\end{array}$ & 204 & 24 & 452 & 31 \\
\hline $\begin{array}{l}\text { Journal of Consumer } \\
\text { Research (3) }\end{array}$ & 157 & 19 & 228 & 16 \\
\hline Marketing Science (6) & 94 & 11 & 126 & 9 \\
\hline Journal of Retailing (2) & 40 & 5 & 50 & 3 \\
\hline $\begin{array}{l}\text { Journal of Consumer } \\
\text { Psychology (5) }\end{array}$ & 28 & 3 & 35 & 2 \\
\hline $\begin{array}{l}\text { Journal of Interactive } \\
\text { Marketing (7) }\end{array}$ & 23 & 3 & 24 & 2 \\
\hline $\begin{array}{l}\text { Journal of Public Policy } \\
\text { \& Marketing (10) }\end{array}$ & 17 & 2 & 24 & 2 \\
\hline $\begin{array}{l}\text { Journal of Service } \\
\text { Research (8) }\end{array}$ & 14 & 2 & 17 & 1 \\
\hline $\begin{array}{l}\text { Journal of Consumer } \\
\text { Affairs (9) }\end{array}$ & 6 & 1 & 6 & 0 \\
\hline Total & 838 & 100 & 1.436 & 100 \\
\hline
\end{tabular}

Fonte: Elaborada pelos autores deste artigo
Nota-se que há maior referência a artigos de marketing pertencentes ao Journal of Marketing (JM), o que é verificado tanto em termos de diferentes artigos como no geral. Esses resultados corroboram os achados de Baumgartner e Pieters (2003) que evidenciam a amplitude da influência interna e externa do JM. Em seguida estão os artigos do Journal of Marketing Research (JMR) e do Journal of Consumer Research (JCR). Juntos, os artigos desses três periódicos representam $73 \%$ dos diferentes artigos de marketing referenciados e $80 \%$ do número total de artigos referenciados.

Os dez artigos mais referenciados, agrupados, representam $13 \%$ do total de referências a trabalhos de marketing. Entre esses artigos há predominância daqueles publicados no JMR, o que evidencia um tipo de diálogo não necessariamente atrelado às questões teóricas do campo, mas sim ao desenvolvimento metodológico. Com efeito, sete dos dez artigos mais referenciados na área de negócios tratam de questões metodológicas. Uma possível explicação para isso é a qualidade da discussão sobre método desencadeada no âmbito da disciplina, especialmente no JMR, cuja orientação positivista é reconhecida. Na medida em que tais discussões sobre método embasam decisões dos pesquisadores da área de negócios especula-se que, também, os principais periódicos da área privilegiem pesquisas de natureza positivista.

Se por um lado a lista dos principais artigos destaca a qualidade e a relevância da discussão feita em torno do aperfeiçoamento dos métodos, modelos e técnicas de coleta e análise de dados, ela denuncia a relativa perda de importância da discussão teórica e dos temas de pesquisa empírica que se desenrolam nos periódicos de marketing. Apesar de as pesquisas de marketing abrangerem um escopo bastante ampliado, como o que se viu em discussão anterior, poucos parecem ser os temas que interessam ou que rendem pesquisas passíveis de ganhar eco na área de negócios. Esses poucos temas são marketing de relacionamento, orientação para mercado e responsabilidade social. Tal achado vai ao encontro das preocupações de MacInnis (2005) em relação ao foco em metodologia, o qual seria um dos responsáveis por minar a relevância teórica da área.

A análise das referências e das citações realizadas pelos autores da área de negócios evidencia um diálogo aberto com a área de marketing, contrariando 
alguns alertas sobre a suposta falta de interação entre as duas (BAUMGARTNER; PIETERS, 2003). Ademais, verificou-se que marketing possui uma influência importante junto à área de negócios. Contudo, apesar de não ser restrita, essa influência parece ser maior para os artigos que se dedicam a debater questões metodológicas em detrimento das discussões teóricas ocorridas no âmbito do marketing, ressaltando um dos efeitos colaterais do excessivo viés metodológico da área de marketing. (MACINNIS, 2005)

\subsection{A Influência de Marketing na Área de Comunicação}

A comunicação é outra área de considerável contato com o marketing, de acordo com o levantamento realizado. A Tabela 3 apresenta a quantidade e o percentual relativo de artigos que fazem referência a textos de marketing, bem como a quantidade de citações a esses trabalhos. Assim como na área de negócios, grande concentração das referências e citações em poucos periódicos. De fato, os três principais periódicos citados representam $78 \%$ das referências encontradas e $79 \%$ do total de citações dos artigos. Destaca-se na área de comunicação a posição do JCR como o principal periódico de marketing a influenciar os trabalhos dos autores, o que confirma a influência da área de comportamento do consumidor no campo da comunicação.

Os dez principais artigos representam 9\% do total de artigos de marketing referenciados pela área de comunicação, o que demonstra uma menor concentração da influência de poucos trabalhos em relação à área anteriormente analisada. Essa diversidade é verificada, igualmente, nos temas dos artigos mais referenciados. Apenas um deles refere-se a discussões sobre método, já o restante trata diferentes temas pesquisados especialmente em comportamento do consumidor, os quais encontram eco nos estudos de comunicação. Tais trabalhos ocupam-se de analisar e mensurar construtos ou fatores que venham a contribuir especialmente para a eficácia de propagandas, com um foco gerencial proeminente. Trata-se de temas efetivamente de fronteira entre a área do marketing e da comunicação, em que a primeira, possivelmente mais uma vez pela qualidade das pesquisas que desenvolve, tem conseguido influenciar escolhas teóricas realizadas no âmbito da segunda.
Tabela 3: Quantidade de artigos de marketing referenciados em comunicação

\begin{tabular}{|c|c|c|c|c|}
\hline \multirow{2}{*}{$\begin{array}{l}\text { PeRIódicos de MARKETING } \\
\text { (RANKING FI } 5 \text { ANos) }\end{array}$} & \multicolumn{2}{|c|}{$\begin{array}{c}\text { Artigos } \\
\text { DIFERENTES }\end{array}$} & \multicolumn{2}{|c|}{ Total } \\
\hline & $\mathbf{N}$ & $\%$ & $\mathbf{N}$ & $\%$ \\
\hline $\begin{array}{l}\text { Journal of Consumer } \\
\text { Research (3) }\end{array}$ & 148 & 38 & 190 & 38 \\
\hline Journal of Marketing (1) & 81 & 21 & 113 & 22 \\
\hline $\begin{array}{l}\text { Journal of Marketing } \\
\text { Research (4) }\end{array}$ & 73 & 19 & 97 & 19 \\
\hline $\begin{array}{l}\text { Journal of Consumer } \\
\text { Psychology (5) }\end{array}$ & 20 & 5 & 24 & 5 \\
\hline $\begin{array}{l}\text { Journal of Interactive } \\
\text { Marketing (7) }\end{array}$ & 17 & 4 & 17 & 3 \\
\hline Marketing Science (6) & 15 & 4 & 20 & 4 \\
\hline Journal of Consumer Affairs (9) & 13 & 3 & 14 & 3 \\
\hline $\begin{array}{l}\text { Journal of Public Policy } \\
\text { \& Marketing (10) }\end{array}$ & 11 & 3 & 12 & 2 \\
\hline Journal of Retailing (2) & 10 & 3 & 14 & 3 \\
\hline Journal of Service Research (8) & 3 & 1 & 3 & 1 \\
\hline Total & 391 & 100 & 504 & 100 \\
\hline
\end{tabular}

Fonte: Elaborada pelos autores deste artigo

Apesar de apresentar uma quantidade consideravelmente menor de referências que aquela verificada na área de negócios, o que evidencia uma influência menor do marketing, a análise de citações da área de comunicação dá conta de uma importante contribuição da escola de comportamento do consumidor. Essa análise demonstra a existência de uma intersecção de interesses entre as duas áreas, com uma contribuição aparentemente importante dos trabalhos, em especial do JCR, em alguns assuntos de comunicação, notadamente aqueles com maiores implicações gerenciais.

\subsection{A Influência de Marketing na Área de Psicologia}

A área de psicologia apresenta uma contribuição histórica para o marketing, notadamente para a escola de comportamento do consumidor (WILKIE; MOORE, 2003). Contudo, os dados demonstram que a recíproca se mostra limitada a poucos trabalhos, em especial àqueles da área de comportamento do consumidor, como verificado na Tabela 4. 
Tabela 4: Quantidade de artigos de marketing referenciados em psicologia

\begin{tabular}{|c|c|c|c|c|}
\hline \multirow{2}{*}{$\begin{array}{c}\text { Periódicos de MARKeting } \\
\text { (RANKING FI } 5 \text { ANos) }\end{array}$} & \multicolumn{2}{|c|}{$\begin{array}{c}\text { Artigos } \\
\text { DIFERENTES }\end{array}$} & \multicolumn{2}{|c|}{ Total } \\
\hline & $\mathbf{N}$ & $\%$ & $\mathbf{N}$ & $\%$ \\
\hline Journal of Consumer Research (3) & 85 & 60 & 93 & 57 \\
\hline Journal of Marketing Research (4) & 29 & 20 & 37 & 23 \\
\hline $\begin{array}{l}\text { Journal of Consumer } \\
\text { Psychology (5) }\end{array}$ & 16 & 11 & 20 & 12 \\
\hline Journal of Marketing (1) & 5 & 4 & 5 & 3 \\
\hline Marketing Science (6) & 3 & 2 & 3 & 2 \\
\hline $\begin{array}{l}\text { Journal of Interactive } \\
\text { Marketing (7) }\end{array}$ & 2 & 1 & 2 & 1 \\
\hline $\begin{array}{l}\text { Journal of Public Policy } \\
\text { \& Marketing (10) }\end{array}$ & 2 & 1 & 2 & 1 \\
\hline Journal of Retailing (2) & 0 & 0 & 0 & 0 \\
\hline Journal of Service Research (8) & 0 & 0 & 0 & 0 \\
\hline Journal of Consumer Affairs (9) & 0 & 0 & 0 & 0 \\
\hline Total & 142 & 100 & 162 & 100 \\
\hline
\end{tabular}

Fonte: Elaborada pelos autores deste artigo

Como nas áreas anteriores analisadas, há uma forte concentração das referências em torno de três periódicos, que, juntos, representam $91 \%$ dos diferentes artigos e $92 \%$ das referências encontradas. Porém, ao contrário das áreas anteriores, o JM praticamente não apresentou referências, sendo substituído pelo Journal of Consumer Psychology (JCP), o qual, juntamente com o JCR concentra $71 \%$ dos diferentes artigos referenciados e $69 \%$ do total de referências, o que comprova a preponderância do interesse, ainda que limitado, na área do comportamento do consumidor.

Essa pequena influência da área de marketing deve ser, no entanto, relativizada. Assim como o próprio marketing, o campo da psicologia possui um amplo escopo de interesse, que se reflete na diversidade de artigos publicados em seus periódicos, grande parte deles sem qualquer relação com mercados, trocas ou relações de consumo. É natural, portanto, que um artigo que discuta relações familiares ou trau- ma de infância não busque basear seus argumentos em referências de marketing ou comportamento do consumidor, como também é esperado que um artigo discutindo orientação para mercado não se baseie em referências da psicologia. Assim, importa salientar que em grande parte dos artigos encontrados que discutem consumo na área de psicologia, trabalhos de marketing se fazem presentes. Tal presença evidencia um ponto de intersecção importante entre a psicologia e o comportamento do consumidor.

Os três principais artigos referenciados reúnem $7 \%$ do total de referências encontradas. A pouca concentração de referências aponta para uma variedade de trabalhos utilizados pelos autores de psicologia. A natureza dos artigos mais referenciados também destaca a influência da área de comportamento do consumidor nos estudos de psicologia, notadamente naqueles orientados para compreender aspectos das relações de consumo. Os temas envolvem percepção, experiências e tomada de decisão, destacando-se a ausência de artigos que tratam de metodologia.

Assim como na área de comunicação, a área de psicologia apresenta uma interface importante entre uma subárea da psicologia e o comportamento do consumidor. A análise de citações demonstra a influência desses artigos, especialmente através do JRC, com ênfase para as contribuições teórico-empíricas do marketing.

\subsection{A Influência de Marketing nas Áreas de Economia e Finanças}

A economia é considerada a ciência-mãe do marketing (Bartels, 1988). Dela, foram apropriados diversos conceitos que contribuíram para a formação do corpo teórico da disciplina. No entanto, quando se observa a contribuição do marketing para a economia, por meio dos dados da Tabela 5 , verifica-se que a recíproca não é verdadeira, havendo um número relativamente reduzido de referências a trabalhos do primeiro, o que está de acordo com os resultados encontrados por Pieters e Baumgartner (2002). 
Tabela 5: Quantidade de artigos de marketing referenciados em economia e finanças

\begin{tabular}{|c|c|c|c|c|}
\hline \multirow{2}{*}{$\begin{array}{c}\text { PeRIódicos de MARKETING } \\
\text { (RANKING FI } 5 \text { ANOs) }\end{array}$} & \multicolumn{2}{|c|}{$\begin{array}{c}\text { Artigos } \\
\text { DifERENTES }\end{array}$} & \multicolumn{2}{|c|}{ TOTAL } \\
\hline & $\mathbf{N}$ & $\%$ & $\mathbf{N}$ & $\%$ \\
\hline Journal of Consumer Research (3) & 21 & 34 & 22 & 33 \\
\hline Journal of Marketing Research (4) & 18 & 30 & 19 & 29 \\
\hline Marketing Science (6) & 14 & 23 & 17 & 26 \\
\hline Journal of Marketing (1) & 3 & 5 & 3 & 5 \\
\hline $\begin{array}{l}\text { Journal of Consumer } \\
\text { Psychology (5) }\end{array}$ & 2 & 3 & 2 & 3 \\
\hline Journal of Consumer Affairs (9) & 1 & 2 & 1 & 2 \\
\hline $\begin{array}{l}\text { Journal of Public Policy } \\
\text { \& Marketing (10) }\end{array}$ & 1 & 2 & 1 & 2 \\
\hline Journal of Retailing (2) & 1 & 2 & 1 & 2 \\
\hline $\begin{array}{l}\text { Journal of Interactive } \\
\text { Marketing (7) }\end{array}$ & 0 & 0 & 0 & 0 \\
\hline Journal of Service Research (8) & 0 & 0 & 0 & 0 \\
\hline Total & 61 & 100 & 66 & 100 \\
\hline
\end{tabular}

Fonte: Elaborada pelos autores deste artigo

Assim como nas áreas anteriores analisadas, há uma forte concentração das referências em torno de três periódicos, que juntos representam $87 \%$ dos diferentes artigos e $88 \%$ das referências encontradas. Assim como em psicologia, o JM praticamente não apresentou referências, sendo substituído, nesse caso, pelo Marketing Science que juntamente com o JMR compõem $32 \%$ dos diferentes artigos e $36 \%$ das referências encontradas, o que indica um viés teórico-metodológico da contribuição de marketing para finanças. Ao mesmo tempo, o JCR permanece como o periódico mais citado, indicando mais uma vez a relevância da área de comportamento do consumidor.

Embora haja apenas três artigos com alguma repetição, eles representam $9 \%$ do total de referências. Verifica-se, mais uma vez, o viés de comportamento do consumidor influenciando a área de economia e finanças, na qual preponderam os temas de contabilidade mental, escolha do consumidor e reação a preços. Evidencia-se mais um ponto de intersecção entre comportamento do consumidor e, nesse caso, economia e finanças, em especial microeconomia.

Apesar de apresentar um número limitado de referências da área de marketing, a análise das citações evidencia a existência de um diálogo com a área de comportamento do consumidor, cujas pesquisas teórico-empíricas mostram-se relevantes na composição do argumento de alguns trabalhos de economia. Assim como verificado em relação à área de psicologia, a economia apresenta um amplo escopo de pesquisas em suas publicações, o que permite relativizar as poucas referências encontradas.

\subsection{A Influência de Marketing na Área de Sociologia}

A área de sociologia é uma das principais exportadoras de conhecimento para outras áreas, segundo a análise de Pieters e Baumgartner (2002). A mesma análise evidencia alguma utilização de textos de sociologia na área do marketing e nenhuma de marketing em sociologia. Essa ausência de influência da área de marketing também é verificada na presente pesquisa, onde foram encontradas referências a 32 artigos diferentes da área de marketing.

Em meio à carência de referências da área de marketing destacam-se o JMR (16 artigos diferentes) e o JCR (9), responsáveis por $78 \%$ dos diferentes artigos referenciados e por $68 \%$ do total de referências, o que indica respectivamente um interesse pelo desenvolvimento metodológico e pela área de comportamento do consumidor. Outros periódicos referenciados nos artigos de sociologia analisados são: Journal of Consumer Affairs (JCA) (3), JM (2), JCP (1) e Journal of Interactive Marketing (1).

A análise dos artigos mais referenciados reforça o interesse por alguns aspectos teóricos de comportamento do consumidor, sendo quatro trabalhos, dos cinco citados mais de uma vez, da área de comportamento do consumidor. Diferentemente das demais áreas que referenciam textos de comportamento do consumidor, na sociologia tais referências estão menos ligadas às pesquisas conclusivas, mas sim a aspectos teórico-empíricos substantivos, tais como a análise de questões sociais mais amplas como o acesso a serviços públicos e privados, aos papéis e estruturas familiares $e$ aos padrões de formação e difusão de opinião.

Em seu conjunto, a análise das referências aos textos de marketing reforça a ideia de dois centros de interesse para os sociólogos, sendo o primeiro o aspecto metodológico e o segundo, os desenvolvimentos 
teórico-empíricos acerca de temas substantivos do comportamento do consumidor.

\subsection{A Influência de Marketing na Área de Antropologia}

A aparente endogenia encontrada por Pieters $e$ Baumgartner (2002) em relação à produção científica de antropologia encontra respaldo na presente pesquisa. A área de antropologia foi a que citou o menor número de referências aos periódicos de marketing. Assim como no caso da Psicologia, entre os títulos pesquisados aqui, há diversos trabalhos sem qualquer relação possível com a área de marketing, porém, o número de citações foi muito baixo inclusive nos artigos com algum ponto de contato. Foram apenas 12 artigos citados em seis anos de publicações dos cinco principais periódicos. Entre as poucas referências, mais uma vez destaca-se o JCR como o periódico com a maior quantidade de referências (5), seguido do JMR (3) e do JM (2). Os periódicos Marketing Science e JCA apresentaram uma referência cada, sendo que os demais não apresentaram referências.

Verifica-se, novamente, certo viés para a área de comportamento do consumidor, sendo o único artigo presente mais de uma vez (duas referências) nas pesquisas de antropologia, um trabalho que discute o comportamento de reclamação e os fluxos da reclamação através da organização.

De maneira geral, os resultados demonstram a existência de um diálogo das diferentes áreas com o marketing. Esse diálogo ocorre especialmente nas áreas de negócios e comunicação, ficando enfraquecido nas demais áreas. A seguir, são tecidas as considerações finais, buscando sintetizar os resultados ora encontrados e oferecer uma apreciação crítica sobre a relevância da disciplina acadêmica de marketing, bem como possíveis caminhos para o incremento dessa importância.

\section{Considerações Finais}

A análise bibliométrica evidenciou a existência de três níveis de influência da área de marketing, apresentados em ordem decrescente: no primeiro nível, encontra-se a área de negócios, no segundo nível, a área de comunicação e, no terceiro nível, as demais áreas. Essa influência pode ser vista de forma otimista ou de forma pessimista. Há argumentos para sustentar ambas as visões.

No primeiro nível, a existência de um diálogo com a área maior de negócios vai de encontro aos argumentos de Baumgartner e Pieters (2003) sobre o distanciamento crescente de marketing das áreas de negócios, evidenciando, ao contrário, a existência de temas da primeira que alimentam discussões da segunda. Com efeito, a quantidade de artigos de marketing referenciados e citados ao longo dos textos é significativa. Contudo, verificou-se que essa influência restringe-se, notadamente, às discussões metodológicas conduzidas no centro da disciplina. Então, se por um lado marketing apresenta uma influência quantitativamente importante, por outro, quando se olha a natureza dos artigos que são mais referenciados, tal influência parece limitada.

É possível que ao adotar um viés de método (MACINNIS, 2005), a disciplina de marketing tenha produzido excelentes discussões e evoluções, as quais servem de base para a tomada de decisões de pesquisa em outras áreas. Nesse sentido, pode-se afirmar que a área de marketing tem se consolidado como importante fornecedora de modelos, técnicas e instrumentos metodológicos, notadamente para a área de negócios. Porém, tal consolidação pode ter ocorrido em detrimento da relevância teórico-empírica dos temas próprios da área, tais como gestão estratégica, conforme já alertado por Hunt (2002), que figura apenas como coadjuvante entre as referências mais importantes para gestão.

No segundo nível de influência, representado pela área de comunicação, há uma quantidade significativamente menor de artigos referenciados, indicando uma queda importante na influência da disciplina. Nessa área, a influência mais nítida concentra-se nos estudos de comportamento do consumidor que analisam $e$ mensuram construtos ou fatores que venham a contribuir especialmente para a eficácia de propagandas, com um foco gerencial proeminente. Verifica-se, então, uma influência específica de marketing naqueles artigos que lidam com um tema de comunicação, dentre tantos da área de comunicação, em que é identificada uma importante área de intersecção.

No terceiro nível encontram-se as disciplinas relativamente pouco influenciadas por estudos de marketing. Pode-se dizer que a disciplina apenas ofere- 
ce uma contribuição limitada e pontual. Na psicologia, área que oferece suporte para parte importante dos estudos de comportamento do consumidor (WILKIE; MOORE, 2003), há uma influência apenas limitada, porém bastante marcada pela presença de artigos de comportamento do consumidor. Da mesma forma, em relação à economia, base inicial para os estudos de marketing (BARTELS, 1988), os resultados encontrados indicam uma fraca relação, em linha com os achados de Pieters e Baumgartner (2002). Nesse sentido, as referências de marketing encontradas na área de economia e finanças indicam um viés teóricometodológico da contribuição do marketing, bem como relevância da área de comportamento do consumidor. Se por um lado o fluxo de artigos da área de marketing para essas duas áreas é limitado, o entendimento dessas áreas como áreas de amplo escopo, com consequente variedade de espaço para trabalhos de diversas subáreas, permite relativizar os resultados, identificando a existência de avenidas abertas e de um potencial de contribuição importante da área de marketing para as duas disciplinas, notadamente dos trabalhos ligados ao comportamento do consumidor.

As duas últimas áreas pertencentes ao terceiro nível, sociologia e antropologia, acolhem um número extremamente limitado de textos de marketing. Apesar de a sociologia possuir um fluxo de contribuições importante para área de marketing, a recíproca não se mostrou verdadeira (PIETERS; BAUMGARTNER, 2002). Em sociologia, as escassas referências encontradas denotam um interesse pelo desenvolvimento metodológico do marketing e pelo comportamento do consumidor, o qual está ligado mais a aspectos relacionados a questões sociais amplas. Já em antropologia, uma área que acolhe poucas citações de outras disciplinas (idem), foi constatada uma quase inexistência de referências a marketing, com algum destaque para estudos de comportamento do consumidor. Os números retratam um desinteresse contundente por marketing, ao menos de parte das correntes dominantes nas duas áreas, o que pode ocorrer devido ao desconhecimento ou à falta de relevância da área, talvez por conta de um suposto enfoque gerencial, para esses autores. Esses motivos carecem de uma maior investigação.

Em suma, os resultados demonstram que a disciplina de marketing não fala apenas para si. Contudo, a área ainda parece muito distante de consolidar-se como uma "exportadora" de conhecimento, o que a coloca, de certa forma, em posição de coadjuvante entre as ciências sociais e sociais aplicadas. Porém, na medida em que a disciplina se direciona para a compreensão de domínios tão importantes das sociedades capitalistas, ela não mereceria um lugar mais destacado? Dessa forma, reflete-se sobre como é possível ampliar a relevância de marketing para o aprofundamento do conhecimento em outros campos de estudos. Um caminho que se desenha pertinente seria o de fortificar os estudos de marketing nas áreas em que este se mostrou mais relevante. No entanto, isso implica em assumir algum risco, pois o comportamento do consumidor tem se destacado como uma área que vem, aos poucos, tornando-se independente do marketing, ao passo que o desenvolvimento metodológico não pode ser considerado uma área em si. Outro caminho passaria pela tentativa de redução da lacuna entre teoria e prática e pelo aprimoramento do diálogo com a área de estratégia (HUNT, 2002), compreendendo a essência do marketing, como disciplina que se dedica a estudar as relações de troca economicamente constituídas em contextos de mercado. Isso implicaria na redução do escopo da disciplina? Até que ponto tal redução poderia ser benéfica? Volta-se ao ponto de partida e encerra-se esse estudo com mais perguntas do que respostas.

O presente estudo não se desenvolveu sem limitações. Do ponto de vista das escolhas analíticas, há diversos aspectos que poderiam ter sido levados em conta, tais como a inclusão de palavras-chave dos artigos citados e dos artigos que citam, bem como uma análise mais detalhada dos periódicos das outras áreas e dos próprios artigos que realizam as citações, de modo a compreender o contexto dos periódicos e da própria citação. As conclusões obtidas teriam se beneficiado de um aprofundamento da análise da quantidade de citações por artigo, o que teria permitido maior propriedade nas afirmações em relação à intensidade da relevância dos artigos de marketing citados.

Enfim, o artigo deixa espaço para novas pesquisas, tanto em relação aos resultados aqui encontrados, quanto no que diz respeito à aplicação de técnicas de análise bibliométrica, as quais, como deixam claros os resultados, permitem uma série de revelações sobre a dinâmica da construção do conhecimento científico. 


\section{REFERÊNCIAS}

ANDERSON, M. H. How can we know what we think until we see what we said? A citation and citation context analysis of Karl Weick's The Social Psychology of Organizing. Organization Studies, USA, v. 27, n. 11, p. 1.675-1.692, 2006.

ANDREASEN, A. R. The life trajectory of social marketing: some implications. Marketing Theory, USA, v. 3, n. 3, p. 293-303, 2003.

\section{BARTELS, R. The history of marketing thought.}

Columbus: Publishing Horizons, 1988.

BAUMGARTNER, H.; PIETERS R. The structural influence of marketing journals: a citation analysis of the discipline and its subareas over time. Journal of Marketing, USA, v. 67, n. 2, p. 123-139, 2003.

CASTILHOS, R. B. Apropriações da obra de Pierre Bourdieu no campo do marketing no Brasil. In: ENCONTRO DA ANPAD, 31, 2007, Rio de Janeiro. Anais... Rio de Janeiro: ANPAD, 2007.

DAY, G. S.; MONTGOMERY, D. B. Charting new directions for marketing. Journal of Marketing, USA, v. 63, n. 4, p. 3-13, 1999.

DHOLAKIA, N.; FIRAT, A. F.; BAGOZZI, R. P. The de-americanization of marketing thought: in search of a universal basis. In: LAMB, C. W.; DUNNE, P. (Ed.). Theoretical developments in marketing. Chicago: American Marketing Association, 1980.

FERREIRA, M. P.; SERRA, F. R.; ALMEIRA, M. I. R. DE. Estudo Bibliométrico da Contribuição de Buckley e Casson (1976) na Pesquisa de Negócios Internacionais.

Revista de Ciências da Administração, Florianópolis, v. 14, n. 2012, p. 9-24, 2012.

FULLERTON, R. A. How modern is modern marketing? Marketing's evolution and the myth of the "production era”. Journal of Marketing, USA, v. 52, n. 1, p. 108125, 1988.

HASTINGS, G.; SAREN, M. The critical contribution of social marketing: theory and application. Marketing Theory, USA, v. 3, n. 3, p. 305-322, 2003.
HUNT, S. D. The nature and scope of marketing.

Journal of Marketing, USA, v. 40, n. 3, p. 17-28, 1976.

Foundations of marketing theory: toward a general theory of marketing. Armonk: M. E. Sharpe, 2002.

JONES, D. G. B.; SHAW E. H.; MCLEAN, P. A. The modern schools of marketing thought. In: McLaran, P. et al. Handbook of Marketing Theory. Los Angeles: Sage, 2010.

KEITH, R. The marketing revolution. Journal of Marketing, USA, v. 24, n. 1, p. 35-38, 1960.

KOTLER, P.; LEVY, S. J. A new form of marketing myopia: rejoinder to professor Luck. Journal of Marketing, USA, v. 33, n. 3, p. 55-57, 1969a.

KOTLER, P.; LEVY, S. J. Broadening the concept of marketing. Journal of Marketing, USA, v. 33, n. 1, p. 10-15, $1969 b$.

LUCK, D. Broadening the concept of marketing - too far. Journal of Marketing, USA, v. 33, n. 3, p. 53-54, 1969.

MACINNIS, D. Them versus us: woes on the bifurcation of the academic marketing discipline. In: BOLTON, R. (Org.). Marketing Renaissance: opportunities and imperatives for improving marketing thought, practice, and infrastructure listen and act differently. Journal of Marketing, USA, v. 69, n. 4, p. 1-25, 2005.

MCALISTER, L. Unleashing potential. In: BOLTON, R. (Org.). Marketing Renaissance: opportunities and imperatives for improving marketing thought, practice, and infrastructure listen and act differently. Journal of Marketing, USA, v. 69, n. 4, p. 1-25, 2005.

MCKENNA, R. Marketing is everything. Harvard

Business Review, USA, v. 53, n. 2, p. 2-10, 1991.

MORAN, M. R. et al. Alianças Estratégicas : uma análise bibliométrica da produção científica entre 1989 e 2008.

Revista de Ciências da Administração, Florianópolis, v. 12 , n. 27 , p. $63-85,2010$.

PIERCY, N. F. Research in marketing: teasing with trivia or risking relevance? European Journal of Marketing, USA, v. 36, n. 3, p. 350-363, 2002. 
PIETERS, R.; BAUMGARTNER, H. Who talks to whom? Intra and interdisciplinary communication of economics journals. Journal of Economic Literature, USA, v. 40, n. 2, p. 483-509, 2002.

RIBEIRO, H. C. M. et al. Visão Baseada em Recursos: uma Análise Bibliométrica dos últimos 11 anos. Revista de Ciências da Administração, Florianópolis, v. 14, n. 34, p. 39-59, 2012.

ROSSI, C. A. V. A utilidade da pesquisa do consumidor. In: ENCONTRO DE MARKETING DA ANPAD, 3, 2008, Curitiba. Anais...Curitiba: ANPAD, 2008.

SMALL, H. Cited documents as concept symbols. Social Studies of Science, USA, v. 8, n. 3, p. 327-340, 1987.

SHAW, E. H.; JONES, D. G. A history of schools of marketing thought. Marketing Theory, USA, v. 5, n. 3, p. 239-281, 2005.

SHETH, J.; SISODIA, R. Does marketing need reform? In: Bolton, R. (Org.). Marketing Renaissance: opportunities and imperatives for improving marketing thought, practice, and infrastructure listen and act differently.

Journal of Marketing, USA, v. 69, n. 4, p. 1-25, 2005.

VARGO, S.; LUSCH, R. Evolving to a new dominant logic for marketing. Journal of Marketing, USA, v. 68, n. 1, p. 1-17, 2004.

VIEIRA, V. A. Who is who in marketing academy? What is his/her best paper? How much is it cited? Understanding the long term contribution and productivity from the Brazilian scientists. In: ENCONTRO DE MARKETING DA ANPAD, 4, 2010, Florianópolis. Anais... Florianópolis: ANPAD, 2010.

WALTER, S. A.; ROCHA, D. T. da. A contribuição de Thomas Kuhn para a produção científica em administração. Revista de Ciências da

Administração, Florianópolis, v. 13, n. 30, p. 11-38, 23 ago. 2011.

WILKIE, W.; MOORE, S. Scholarly research in marketing: exploring the " 4 eras" of thought development. Journal of Public Policy \& Marketing, USA, v. 22, n. 2, p. 116146, 2003.

\section{NotAs}

1 Consulta realizada em agosto de 2011.

2 Alguns números do ano de 2011 não haviam sido disponibilizados até a data do encerramento deste artigo. 